\title{
Role of Therapy-Induced Cellular Senescence in Tumor Cells and its Modification in Radiotherapy: The Good, The Bad and The Ugly
}

David Murray* and Razmik Mirzayans

Department of Oncology, Division of Experimental Oncology, University of Alberta, Edmonton, Alberta, Canada

\begin{abstract}
The last decade has seen major advances in the clinical practice of radiotherapy (RT). Our understanding of the biological effects occurring after exposure of cells and tissues to ionizing radiation has also increased enormously in this period. In this article we will summarize our current knowledge and key knowledge gaps in an area that is emerging as a potentially important factor in tumor responses to RT, namely the activation of the therapy-induced cellular senescence (TCS) pathway and its associated secretory response, the so-called senescence-associated secretory phenotype or "SASP". Although the existing literature on these responses is substantial in the chemotherapy domain, the information specific to RT has unfortunately lagged behind. This includes knowledge relating to the factors that govern the ability of tumor cells to switch from TCS to another terminal/irreversible mode of cell death or to escape from TCS and recover proliferative potential. We will therefore examine some of the implications of TCS and SASP from the perspectives of better understanding the biological basis of the various types of RT delivery. We will also consider the implications of this knowledge for the development and use of modifying agents that either reinforce the TCS phenotype and circumvent recovery pathways or switch the cells from TCS into a terminal apoptotic pathway that may represent a more desirable outcome clinically.
\end{abstract}

Keywords: Tumor cells; Radiation therapy

\section{Introduction}

Radiation therapy (RT) in its various forms-external beam RT (EBRT), low dose rate radioisotope and radioimmunotherapy (RIT), and brachytherapy-is a mainstay of cancer treatment. Even in the post-human genome era, which has seen intensive research into molecularly-targeted cancer therapeutics, it is estimated that about $40 \%$ of cancer cures are attributable to RT [1]. Major improvements in RT in the last decade have come largely from technological advances that allow better localization of dose to cancerous tissue based on the use of image guidance. However, this period has also witnessed extraordinary advances in our understanding of the biological effects of ionizing radiation (IR) at the cell and tissue level. RT exerts its anticancer effects primarily by damaging the genomic DNA of tumor cells and by activating anti-tumor immune responses in some circumstances. A staggering amount of knowledge has been generated surrounding the chain of cell- autonomous events that process IR-induced DNA damage, and especially on the resolution of cellular DNA double-strand breaks (DSBs). Cell survival requires that DSBs are rapidly eliminated from the genome. This is the realm of the DNA damage response (DDR), a highly orchestrated molecular network whose function is to detect and repair such DNA lesions $[2,3]$. If this process fails, the cell may be shunted into one of the cell-death pathways described below.

The cell-autonomous component of the DDR was the major picture until quite recently. However, the last decade has witnessed the uncovering of a whole new cell non-autonomous aspect of the DDR that is often referred to as the 'DNA damage secretory program' or DDSP [4]. The DDSP involves the secretion of a broad range of factorsincluding pro-inflammatory chemokines and cytokines, growth and angiogenic factors, and proteases-by multiple cell types (both normal and cancerous) within the irradiated field, and it is emerging as a critical determinant of the response of tumors to RT, including the recruitment of immunological cells to the irradiated tumor site. Many of these secreted DDSP factors are known to impact on sensitivity or resistance to RT. Understanding these events and their relative importance to the various types of RT will be important for developing therapeutic strategies that modify the tumor microenvironment and its interaction with tumor cells [4-18].

\section{Modes of Clonogenic Cell Death Following IR Exposure}

The linear-quadratic (LQ) model that is widely used in RT treatment planning is based on the assumption that the direct killing of tumor cells is the major determinant of tumor control probability. Because the model is based on a binary outcome: clonogenic cell death versus survival; it does not require any consideration of the fact that several different modes of cell death can contribute to the loss of clonogenic potential depending on factors such as cell type and microenvironment as well as IR dose. These modes include apoptosis, autophagy, mitotic catastrophe and necrosis/necroptosis, the key features of which are summarized in Table 1. Information on mode of cell death may be irrelevant for clinical application of the LQ model but is likely a major factor in the failure of predictive assays for RT outcomes that were based on measurements of apoptosis alone $[19,20]$. It is also critical to understand the mode of cell death in a personalized or segmentalized medicine context if we are going to judiciously apply a particular radio modifying agent to an individual patient or group of patients, as will be discussed below.

This review will focus on another pathway that leads to the loss of cellular clonogenic potential after IR exposure: therapy-induced cellular senescence (TCS). This pathway, which is probably a significant factor

*Corresponding author: David Murray, Department of Oncology, Division of Experimental Oncology, University of Alberta, Edmonton, Alberta, Canada E-mail: David.Murray5@albertahealthservices.ca

Received August 23, 2013; Accepted October 18, 2013; Published October 22, 2013

Citation: Murray D, Mirzayans R (2013) Role of Therapy-Induced Cellular Senescence in Tumor Cells and its Modification in Radiotherapy: The Good, The Bad and The Ugly. J Nucl Med Radiat Ther S6: 018. doi:10.4172/2155-9619.S6-018

Copyright: (c) 2013 Onyeuku NE, et al. This is an open-access article distributed under the terms of the Creative Commons Attribution License, which permits unrestricted use, distribution, and reproduction in any medium, provided the original author and source are credited. 
Citation: Murray D, Mirzayans R (2013) Role of Therapy-Induced Cellular Senescence in Tumor Cells and its Modification in Radiotherapy: The Good, The Bad and The Ugly. J Nucl Med Radiat Ther S6: 018. doi:10.4172/2155-9619.S6-018

Page 2 of 7

\begin{tabular}{|c|c|}
\hline Mode & Characteristics \\
\hline Apoptosis & $\begin{array}{l}\text { - A genetically-regulated type of programmed cell death. } \\
\text { - Follows either the intrinsic (mitochondrial) DNA damage-mediated pathway or the extrinsic death-receptor pathway, both of which involve } \\
\text { activation of caspases [5]. } \\
\text { - Involves chromatin/nuclear condensation, non-random DNA degradation ("laddering"), and cell fragmentation generating apoptotic bodies. } \\
\text { - In vascular endothelial cells, IR damage to the cell membrane can activate acid sphingomyelinase which hydrolyzes sphingomyelin to } \\
\text { ceramide, triggering apoptosis [6]. }\end{array}$ \\
\hline Autophagy & $\begin{array}{l}\text { - A genetically-regulated stress response seen in some human cancer cell lines exposed to IR [7-10]. } \\
\text { - Cells exit the cell cycle, develop cytoplasmic vacuoles that sequester organelles such as mitochondria and ribosomes, auto-digest proteins } \\
\text { and damaged organelles and recycle amino and fatty acids. } \\
\text { - Pro-death (versus pro-survival) signals may require hyper-activation of the autophagy pathway [11]. } \\
\text { - Pathway is negatively regulated by the mTOR (mammalian target of rapamycin) protein. }\end{array}$ \\
\hline Mitotic catastrophe & $\begin{array}{l}\text { - The failure of a cell with damaged DNA to undergo proper mitosis [12]. } \\
\text { - Likely caused by aberrant chromosome segregation/cell fusion. } \\
\text { - Cells are typically enlarged with abnormal spindles, micronuclei and de-condensed chromatin; can lead to multinucleated/polyploid giant } \\
\text { cells. } \\
\text { - May be a key contributor to the loss of clonogenic potential in tumor cells and solid tumors exposed to IR, especially those with mutant p53 } \\
\text { [11-13]. } \\
\text { - Mitotic catastrophe may not represent a mode of cell death but rather a process preceding cell death by necrosis, apoptosis, etc. [14]. }\end{array}$ \\
\hline Necrosis & $\begin{array}{l}\text { - Historically regarded as an uncontrolled (i.e., not genetically regulated) form of cell death following high-dose IR exposures that involves } \\
\text { swelling of the cell and mitochondria, disintegration of the cell organelles and membrane, protein denaturation/coagulation and random DNA } \\
\text { degradation [15]. } \\
\text { - A programmed/genetically-regulated type of necrosis, necroptosis, was recently identified [16]. } \\
\text { - IR was reported to induce necroptosis in thyroid and adrenocortical carcinoma cell lines; this response exhibits some overlap with apoptosis } \\
\text { [17]. }\end{array}$ \\
\hline $\begin{array}{l}\text { Therapy-induced cellular } \\
\text { senescence }\end{array}$ & $\begin{array}{l}\text { - A genetically-regulated response to IR-induced DNA damage seen in many solid tumor-derived cell lines, especially those with wild type p53 } \\
\text { [18] although p53-independent and mutant-p53-dependent mechanisms have been described. } \\
\text { - Cells are growth-arrested for extended periods but remain viable and metabolically active, are enlarged and flattened, develop polyploidy } \\
\text { and express the marker "senescence-associated } \beta \text {-galactosidase". } \\
\text { - Important molecular }\end{array}$ \\
\hline
\end{tabular}

Table 1: Key characteristics of the various modes of ionizing radiation- (IR)-induced tumor-cell death.

in tumor-cell responses to RT [13,18,21-23], has been called many things over the last 20 years, including permanent/irreversible growth arrest, stress-induced premature senescence and accelerated cellular senescence. Here we will only use the TCS moniker for clarity. The first of these names may actually be misleading because, as discussed below, it is becoming apparent that some cells that have undergone this putatively "permanent" growth arrest may recover the ability to proliferate at later times while others may transition to one of the other cell-death pathways over time.

\section{Mechanisms of IR-induced TCS}

IR-induced TCS is basically a prolonged exit from the cell division cycle mediated by various cyclin- dependent kinase (CDK) inhibitors such as p21CIP1/WAF1 (CDKN1A), p16INK4a, p19INK4D (Arf) and p27Kip1 (hereafter p21, p16, p19 and p27). The early events that follow irradiation of tumor cells with wild-type (wt) p53 are relatively consistent. The $\mathrm{p} 21$ gene is rapidly transcriptionally activated by $\mathrm{p} 53$; those cells destined to undergo TCS also typically show a delayed wave of sustained nuclear p21 induction (e.g., $[18,24])$. In addition to inhibiting CDKs, p21 also appears to drive TCS by positively regulating senescence genes and negatively regulating mitosis and apoptosis genes/ proteins and by inhibiting proliferating cell nuclear antigen (PCNA), a processivity factor for DNA polymerase $\delta$ in genomic DNA replication $[25,26]$. In cells with p53 mutations and alterations in CDK inhibitors that characterize many human tumors, such life/death decisions in relation to TCS are not so straightforward but will be critical to understand going forward. To illustrate this point, we [27] compared TCS in 4 human cancer cell lines: MDA-MB435s and SKMEL-28 which have mutant p53 (p53-mut) and express p16 (albeit of unknown functional status); MCF-7 which is p53-wt but p16-suppressed; and MDD2 which is derived from MCF7 by transfection with a dominant negative mutant p53 and is p16-suppressed. Surprisingly, for all 4 cell lines exposure to $\gamma$-radiation ( 8 Gy) resulted in TCS in $40 \%$ or more of the cells after 7 days, and this response correlated closely with an increase in the level of p21 but not of p16 (Figure 1). However, p16dependent TCS has been reported to be triggered by IR in some genetic backgrounds, e.g., in p53-mut/p16-proficient non-small cell lung cancer (NSCLC) cell lines such as ABC-1 and HCC44 (supplemental data in [28]). We clearly need greater clarity in regard to such effects and the pathways that drive p53-independent or p53-mut-dependent TCS.

In a given irradiated biological system, indeed even within a culture of single cell line, cells can be progressing along different death pathways. In fact, these mode-of-death commitments can shift with time, such that a tumor cell undergoing IR-induced mitotic catastrophe can progress to an apoptotic state $[19,29,30]$ or to a necrotic state [31]. There are also elements of mechanistic overlap between apoptosis and necroptosis [17] and of functional overlap between TCS and autophagy [32]. As discussed in section 7, the motivation for a tumor cell to progress down one death pathway or another can also be influenced using small-molecule inhibitors that have the potential to be developed into therapeutic drugs.

A key feature of TCS that distinguishes it from other modes of cell death is that the cells are not rapidly deleted from the tumor but instead persist and retain metabolic and secretory activity even though they do not undergo cell division. Indeed a unique idiom-the senescenceassociated secretory phenotype or "SASP"-has been coined by Campisi et al. [33] to distinguish this particular facet of the DDSP. Largely because of SASP, cells in TCS will likely have a continuing impact on therapy outcomes, especially in RIT or fractionated RT regimens where dose increments delivered later in the course of treatment would be superimposed on a tumor in which many of the cells will already be in TCS and thus actively engaged in secretory activity. We will now briefly consider the key knowledge gaps in regard to the TCS phenotype, such as its long-term sustainability versus reversibility and clinical relevance. 


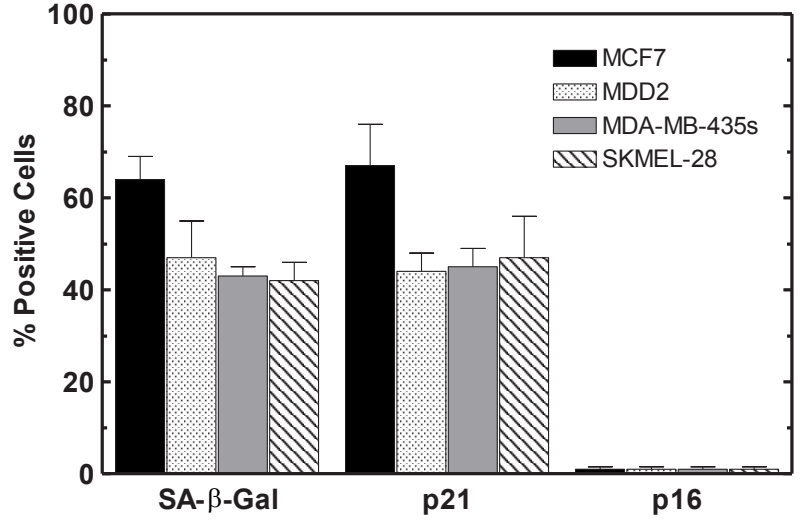

Figure 1: Relationship between the percentages of senescence-associated $\beta$-galactosidase (SA- $\beta$-gal)- positive cells, p21-expressing cells, and p16expressing cells measured in 4 human cancer cell lines at seven days after exposure to an 8-Gy dose of 60Co $\gamma$ radiation. All values for irradiated cultures were corrected by subtracting those for sham-irradiated control cultures. Bars, SE. Adapted from Mirzayans et al. [27]

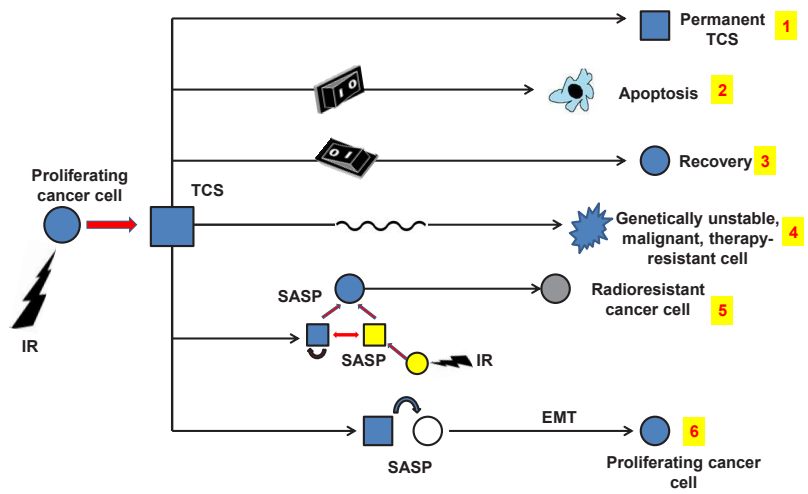

Figure 2: Cartoon illustrating some of the potential long-term fates of a tumor cell after undergoing ionizing radiation- (IR)-induced TCS. Cancer cells in TCS (blue square) can potentially: [1] remain in a permanently arrested state of TCS; [2] transition to a terminal/irreversible cell death pathway such as delayed apoptosis or autophagy; [3] eventually escape from TCS and recover the ability to proliferate (blue circle); [4] develop genetic instability, eventually generating highly malignant and/or therapy-resistant "giant" cells; [5] exert in-trans SASP-mediated effects on proliferating cancer cells, imparting a radioresistant phenotype on those cells (grey circle); such SASP-mediated effects can also be generated or reinforced by normal cells such as fibroblasts within the tumor microenvironment that have undergone IR-induced TCS, illustrated by the yellow circle/square (proliferating/TCS fibroblast); or [6] promote epithelial-mesenchymal transition (EMT) in normal cells (white circle) through their SASP secretion activity and thus drive the genesis of malignancy.

\section{Is IR-Induced TCS a Good or Bad Thing for RT Outcomes?}

Opinion on the contribution of TCS to therapeutic outcomes is evolving along with our knowledge of its mechanism. Initially, inducing TCS in tumor cells was considered to represent a cytostatic mechanism and as such was expected to contribute to short-term tumor control. Although TCS may indeed contribute to the early tumor growth inhibition following RT, from the longer-term perspectives of tumor control and recurrence there remain a number of outstanding issues, as outlined in Figure 2.
First and foremost, a therapeutically advantageous outcome might be achieved if TCS was truly "permanent" or could switch to a terminal cell-death mode such as apoptosis. A key question, though, is whether a subset of these cells might eventually recover their growth potential and repopulate the tumor, leading to tumor recurrence? Some evidence from cell line studies does suggest such a recovery mechanism. Exposing p53-wt MCF-7 breast cancer cells to 10 Gy of IR initially resulted in high levels of TCS, but after about 12 days a proliferating population of cells emerged; no such recovery was seen when p53 was attenuated or mutated [34]. A similar pattern of recovery was seen in MCF-7 cells after exposure to a $2 \mathrm{~Gy} \times 5$ fractionated IR schedule [35]. Several other studies support the premise that a subset of tumor cells can overcome TCS induced by RT or DNA-damaging chemotherapy drugs and regain clonogenic potential [36-43].

An equal if not greater concern is that in several of the studies listed in 4.1 the emergent cells were often characterized as genetically unstable and potentially more malignant and/or resistant to subsequent therapy. Such processes may actually be ongoing in cells that superficially appear to be in a "static" state of TCS.

Even cells in prolonged TCS could represent a significant barrier to therapy because their ongoing SASP means that they can continue to secrete radioprotective factors as well as growth, angiogenic and other factors with the potential to drive the growth and progression of surviving tumor cells [4]. This could be particularly problematic in conventional EBRT schedules in which multiple daily fractions of $\sim 2$ Gy are given over a period of weeks or in low-dose-rate RIT (which involves continued decay of radionuclides) because tumor cells that survive until relatively late in the course of therapy will likely experience high levels of SASP- derived factors secreted by either irradiated tumor cells or normal cells, and thus become more resistant to killing by the subsequent doses. The potential of such SASP signals to protect tumor cells is poignantly illustrated by the demonstration that irradiated fibroblasts that underwent TCS could enhance the proliferation and radioresistance of breast cancer cells with which they were co-cultured [44]. It is possible that some of these negative effects of TCS may be less relevant in the setting of stereotactic ablative RT using a small number of high-dose fractions, and especially in single-dose RT where only one very high dose fraction is given. Whether this will in fact be the case should become evident as we learn more about the underlying biology of these novel RT techniques.

Because of their SASP activity, cells in a state of prolonged TCS induced by DNA-damaging agents may actually promote epithelialmesenchymal transition (EMT) and thus drive the genesis of malignancy [45-47].

The 'bottom line' here is that there are some significant concerns about what might happen to cells in TCS at later times and that it would probably be therapeutically advantageous to find ways of switching cells in TCS (or that would be likely to undergo this response) into a terminal cell-death pathway such as apoptosis, rather than running the risk of cell proliferation recovery or one of these other undesirable outcomes. An alternative strategy would be to develop biomarkers to identify patients at risk for TCS-associated adverse events.

\section{What Factors Determine Whether TCS Is Prolonged or Reversible?}

These mechanisms are not well understood for IR/RT. An obvious question is whether the duration and/or sequence of induction of the various CDK inhibitors are a factor here. Although p21 elevation is 
clearly the key aspect of the early trigger for TCS in many tumor cell lines, this may not be the case in all cell backgrounds; furthermore, the p21 signal is not necessarily prolonged and could be superseded by induction of other CDK inhibitors such as p16, p19 or p27 to maintain the TCS state. Thus it was suggested by Jones et al. [34] that the proliferation recovery after TCS seen in irradiated MCF-7 cultures, which are p53-wt but p16- suppressed, could reflect their lack of functional p16. Indeed we also found that MCF-7 cells showed extensive levels of IR-induced TCS and that their p21 levels increase greatly over the first few days after irradiation (Figure 1), but it would be informative to follow these responses for longer times in individual cells. Another cellular factor implicated in the induction and maintenance of TCS induced by various stressors and DNA-damaging agents, including IR, is the secondary generation of reactive oxygen species (ROS) which has in turn been linked to the induction of p21; in fact, both events may be necessary for maintaining the TCS phenotype involving a feedback loop between ROS and p21 [48,49].

A number of proteins implicated in the maintenance versus reversal of the TCS phenotype after chemotherapy have been elegantly described elsewhere [41] and might offer some insight into mechanisms of relevance to IR effects. These include: (i) the cyclin B1/CDK1 complex, which must be suppressed by CDK inhibitors such as p21 and p27 if p53-mut/null tumor cells are to remain arrested in G2-phase; (ii) levels of these very same CDK inhibitors; (iii) the NIMA-related mitotic kinase Nek6 which blocks TCS by preventing the suppression of CDK1, as does another protein kinase, MKK7; and (iii) survivin, an inhibitor of apoptosis (IAP) family member that is phosphorylated by CDK1, and which is up-regulated in some cancer cells that recover from chemotherapy-induced TCS [50]. All of these molecules need to be systematically evaluated in the context of IR-induced TCS, preferably in isogenic tumor-cell backgrounds with differing p53 and CDK-inhibitor status. Another factor that needs to be better characterized in the context of responses to IR/RT is the communication between TCS and other cell-death pathways such as autophagy that have been identified to influence TCS maintenance versus reversal [39].

\section{Incidence of IR-Induced TCS in vivo}

There is an extensive literature on IR-induced apoptosis in both model systems and the clinical setting; however, as noted above, this represents only one part of a bigger picture of tumor-cell death. IRinduced TCS occurs in many cultured cancer cell lines, as mentioned throughout this review, as well as in cancer xenograft models $[28,49]$. Only recently has data begun to emerge showing that TCS occurs in some tumor cell types. Wu et al. [41] have recently reviewed the literature relating to chemotherapy-induced TCS and concluded that it is a prominent response of solid tumors to anticancer drugs that may contribute significantly to early treatment responses. The chemotherapy-TCS literature is much more mature than that for RT, but we suspect that there will be some commonality as well as some key differences; it might, therefore, provide some good pointers. We will illustrate the current state of knowledge for RT and its limitations by reference to two studies, one on prostate cancer and one on head and neck squamous cell carcinoma (HNSCC).

Two earlier in-vitro studies indicated that TCS is the major mode of cell death in irradiated human prostate cancer cell lines [51,52]. In the former study TCS was associated with long-term expression of both the p21 and p16 genes. On the basis of such reports, Supiot and colleagues at the Princess Margaret Hospital in Toronto [23] examined 11 prostate cancer patients who were treated with hypo-fractionated RT (25 Gy in 5 fractions) prior to surgery. All of these tumors lacked p53 core domain mutations. Whereas no apoptosis was seen in the tumor tissue at the completion of RT, extensive TCS was evident based on biomarkers such as high levels of p21 and low levels of proliferation-associated MIB-1; p16 levels were not obviously increased in these tumors.

The second study, from the MD Anderson Cancer Center in Houston, examined tumor cell lines established from 38 patients with HNSCC who had received EBRT after surgery [49]. Loco-regional recurrence in these patients was strongly associated with disruptive p53 mutations which, in the in-vitro cell lines, was in turn associated with: (i) radioresistance in the clonogenic survival assay; (ii) a decreased susceptibility to undergo IR-induced TCS based on SA- $\beta$-gal staining; (iii) minimal induction of p21 after IR exposure; and (iv) decreased secondary (i.e., prolonged) ROS generation. In marked contrast, these p53 mutations did not affect IR-induced mitotic catastrophe (micronuclei) or apoptosis (which was only seen in a few percent of the cells). Thus, IR-induced TCS in these cell lines was predictive of a positive treatment outcome in the clinic. Although RT-induced TCS obviously could not be measured in this post-surgical RT setting, the implication of this correlation is that TCS very likely does occur in these tumors irradiated in situ.

If we consider the chemotherapy literature in this regard we see some striking findings. Thus, $\mathrm{Wu}$ and colleagues [41] performed a pilot study on 18 patients receiving neoadjuvant chemotherapy or radiochemotherapy for locally advanced NSCLC. They observed decreased overall survival among patients whose tumors had the highest TCS biomarker levels. In this small cohort TCS was therefore associated with disease recurrence and adverse treatment outcome. The negative prognostic effect of TCS was also seen in an exploratory study of 26 patients who were treated with platinum-based neoadjuvant chemotherapy for malignant pleural mesothelioma [53]. Such findings, if validated in larger patient cohorts, would presumably reflect a dominance in these study populations or cancer types of the negative aspects of TCS that can result in tumor recurrence, as was discussed in section 4 .

\section{Manipulation of the IR-Induced TCS and SASP Responses to Improve the Therapeutic Index in RT}

Inducing apoptosis in tumor cells with RT is intuitively attractive from the therapeutic perspective because, in contrast to TCS, the cell is rapidly eliminated and should play no further biological role in the treatment outcome (other than perhaps in the generation of tumor antigens in the context of invoking anti-tumor immune responses). TCS on the other hand, as outlined in section 4 , might initially contribute to tumor growth inhibition but could have negative consequences later on because of recovery of proliferation or because of the cells' active secretory status or genetic instability.

This paradox has led to the search for inhibitors that might switch cells from TCS into an apoptotic pathway, an approach that draws impetus from the fact that this transition can occur naturally. A useful drug would either lower the apoptotic threshold when given before RT, such that a tumor cell that would otherwise have undergone TCS would now directly enter an apoptosis pathway, or it would transition a cell that has already undergone TCS or is in the process of recovery from TCS into an apoptotic response. An example of a drug that might lower the threshold for the TCS to apoptosis transition is EB 1089 (seocalcitol), an analog of vitamin D3 that, when given prior to fractionated exposure of MCF- 7 cultures to $5 \times 2$ Gy of $\gamma$ radiation, enhanced cellular susceptibility to undergo apoptosis while delaying the development 
of TCS and of the subsequent recovery of proliferative capacity [35]. Inhibitors of survivin might also prove useful in this regard considering that survivin knockdown with siRNA or treatment with a TAT-survivin peptide that inhibits CDK1- mediated phosphorylation of survivin can switch tumor cells recovering from TCS into apoptosis [50]. The same concept should apply to the use of pharmacologic inhibitors of CDK1 such as flavopiridol (Alvocidib) which is now undergoing clinical trials, and to statins (HMG-CoA reductase inhibitors) which down-regulate several components of the CDK1 pathway including survivin [41]. Another approach might be to lower the apoptotic threshold of the tumor cells by targeting anti-apoptotic genes/proteins such as BCL-2 or Bcl-XL [54].

Several agents have been suggested or described to act as radiosensitizers via the opposite mechanism, i.e., by lowering the tumor-cell dose threshold for undergoing TCS. These include demethylating and differentiating agents as well as inhibitors of histone deacetylases, BMI-1 and receptor tyrosine kinases. In p53- wt A549 NSCLC cells, radiosensitization by the EGFR antagonists erlotinib and cetuximab was associated with prolonged induction of $\mathrm{p} 21$ and greatly enhanced levels of TCS, but not of apoptosis, both in vitro and in a xenograft model [28]. Surprisingly, in several other NSCLC cell lines that were radiosensitized by these inhibitors, enhancement of TCS was the dominant mechanism not only in p53-wt cells but also in p53-mut cells; in these p53-mut cells, TCS was associated with the induction of p16. Radiosensitization and TCS were also strongly correlated with elevated levels of unrejoined DSBs at $24 \mathrm{~h}$ after irradiation independent of the cells' p53 or p16 status.

An intriguing group of tumor-cell radiosensitizers are the nutlins, which are MDM2 inhibitors that can activate endogenous wt p53 and appear to enhance the propensity of irradiated cells to undergo TCS. For example, treatment of two human NSCLC cell lines-A549 and H460with nutlin-3a enhanced IR-induced tumor cell killing and TCS [55]. This TCS-associated radiosensitization was accompanied by strong activation of the p53-p21 signaling pathway. No apoptosis was seen in these cells. Nutlin-3 was also seen to radiosensitize prostate cancer cells with functional p53 through a mechanism involving the induction of p53-dependent TCS [52]. Nutlin-3 also radiosensitized p53-wt (but not p53-mut) laryngeal squamous cell carcinoma cells in the clonogenic survival assay; again, this effect was correlated with increased TCS but not apoptosis [56].

Another compound that appears to radiosensitize tumor cells by enhancing TCS is the mitochondrial drug metformin, a clinically safe anti-diabetic agent. Skinner et al. [49] reported that metformin selectively radiosensitized HNSCC cell lines with disruptive p53 mutations in part or whole by enhancing TCS. Combining metformin and RT also greatly enhanced tumor growth inhibition in the HN31 orthotopic xenograft model of disruptive p53-mut HNSCC. That such effects might have clinical significance was suggested by the observation that, within a cohort of HNSCC patients of known p53 status treated with postoperative RT, locoregional recurrence was greatly decreased among patients taking metformin (although there were only 10 patients in the metformin group).

Another strategy for tumor radiosensitization involves targeting elements of the DDSP/SASP by inhibiting either specific resistanceassociated secreted factors or the upstream regulators of DDR signaling such as p38 MAP kinase and NF- $\mathrm{KB}$ [4]. In an example of the former approach, analysis of SASP proteins secreted by irradiated MCF-7 cells identified the Raf kinase inhibitor protein (RKIP) as an important enhancer of cell proliferation, invasion and migration and as a potential target for preventing the negative effects of the IR-induced TCS/SASP response [57].

An interesting approach to tumor radiosensitization [58] involves the use of SASP agonists to enhance bystander effects, the phenomenon whereby an irradiated cell transmits signals to non-irradiated cells that invoke manifestations of IR effects in the latter cells. Bystander effects usually result in greater cytotoxicity than would be anticipated on the basis of the LQ model and may contribute to RT outcomes after low-dose-rate RIT [59] or high dose RT when the dose distribution is non-homogeneous, such as in microbeam or grid therapy $[60,61]$. A cautionary note, however, is that bystander signals generated by radiopharmaceuticals [62] and by SASP-mediated events [58] can either inhibit or stimulate tumor-cell growth.

An important area that we will not consider here is the potential for exploiting the communication and synergism between local RTmediated tumor cell death/secretory responses and the host immune system, and specifically the potential for engaging anti-tumor specific immune responses; such events have been expertly reviewed elsewhere by Golden and colleagues [11].

\section{Summary and Future Directions}

Activating the TCS phenotype in tumor cells with RT could clearly have good (long-term growth arrest, spontaneous transition to irreversible cell death), bad (recovery from TCS), or ugly (emergence of highly malignant and/or therapy resistant tumor cells), consequences in a given patient. Understanding the circumstances under which these different longer-term responses are triggered will be important for optimizing the use of RT as it moves into the era of personalized or segmentalized medicine. Identifying which modes of RT-induced cell death are operative in a given tumor type or patient may also be critical in directing treatment decisions as well as in the rational integration of radiomodifying agents.

The observation that robust activation of TCS in tumor cells might identify a subset of HNSCC patients who will have superior responses to post-surgical EBRT [49] indicates that the optimal implementation of this knowledge will require the use of validated biomarkers. A problem that must be addressed with some urgency is the invasive nature of current biomarkers of TCS that necessitate the collection of surgical samples. The development of non-invasive molecular-imaging techniques for TCS involving PET radiotracers in particular will be vital for its longitudinal assessment in individual patients [63]. A similar knowledge gap for RT exists with respect to the positive versus negative prognostic aspects of TCS apparent in the various studies discussed above and for which the existing chemotherapy literature is rather alarming, albeit rather hypothesis-generating at this time. We have even less data to go on for RT. It may turn out that RT and chemotherapy simply have very different implications for the fate of tumor cells that initially undergo TCS. Whether TCS in tumor cells will be associated with better relative outcomes in patients receiving RT than chemotherapy and/or whether the tumor type is a key factor here are important questions to address in larger cohorts of patients and with non- invasive end-points for TCS that can extend our knowledge to treatment schedules other than pre-operative RT.

A number of drugs have been identified that appear to radiosensitize tumor cells by enhancing their propensity to undergo TCS. The potential of metformin to impact on HNSCC patients whose tumors have disruptive p53 mutations through such a mechanism [49] is very encouraging. The nagging question is, is this going to be a good outcome 
Citation: Murray D, Mirzayans R (2013) Role of Therapy-Induced Cellular Senescence in Tumor Cells and its Modification in Radiotherapy: The Good, The Bad and The Ugly. J Nucl Med Radiat Ther S6: 018. doi:10.4172/2155-9619.S6-018

Page 6 of 7

in all patients or tumor types? What is the risk for bad or ugly responses to occur later on? If we are going to follow this path then biomarkers of good, bad and ugly outcomes will clearly be needed. Perhaps the less risky strategy is the one focused on transitioning cells that are either in TCS or about to emerge from it into an irreversible mode of death. Only time and additional high-quality studies will tell. Until then, it remains chillingly apparent that "the only good cancer cell is a dead cancer cell", to quote the former AACR president, Dr. Don Coffey [64].

\section{Acknowledgements}

This work was supported by the Canadian Breast Cancer Foundation-Prairies/ NWT region and by the Alberta Cancer Foundation Donor Designated Gran Courtesy of Mr. Wayne Stanton.

\section{References}

1. The Royal College of Radiologists (2003) Equipment, Workload and Staffing for Radiotherapy in the UK 1997-2002. Royal College of Radiologists, London.

2. Thompson LH (2012) Recognition, signaling, and repair of DNA double-strand breaks produced by ionizing radiation in mammalian cells: the molecular choreography. Mutat Res 751: 158-246.

3. Gospodinov A, Herceg Z (2013) Chromatin structure in double strand break repair. DNA Repair (Amst) 12: 800-810.

4. Sun Y, Nelson PS (2012) Molecular pathways: involving microenvironment damage responses in cancer therapy resistance. Clin Cancer Res 18: 40194025 .

5. Vangestel C, Van de Wiele C, Mees G, Peeters M (2009) Forcing cancer cells to commit suicide. Cancer Biother Radiopharm 24: 395-407.

6. Corre I, Niaudet C, Paris F (2010) Plasma membrane signaling induced by ionizing radiation. Mutat Res 704: 61-67.

7. Ito H, Daido S, Kanzawa T, Kondo S, Kondo Y (2005) Radiation-induced autophagy is associated with LC3 and its inhibition sensitizes malignant glioma cells. Int J Oncol 26: 1401-1410.

8. Daido S, Yamamoto A, Fujiwara K, Sawaya R, Kondo S, et al. (2005) Inhibition of the DNA-dependent protein kinase catalytic subunit radiosensitizes malignant glioma cells by inducing autophagy. Cancer Res 65: 4368-4375.

9. Li Y, Zhang J, Chen X, Liu T, He W, et al. (2012) Molecular machinery of autophagy and its implication in cancer. Am J Med Sci 343: 155-161.

10. Paglin S, Yahalom J (2006) Pathways that regulate autophagy and their role in mediating tumor response to treatment. Autophagy 2: 291-293.

11. Golden EB, Pellicciotta I, Demaria S, Barcellos-Hoff MH, Formenti SC (2012) The convergence of radiation and immunogenic cell death signaling pathways. Front Oncol 2: 88.

12. Ianzini F, Bertoldo A, Kosmacek EA, Phillips SL, Mackey MA (2006) Lack of p53 function promotes radiation-induced mitotic catastrophe in mouse embryonic fibroblast cells. Cancer Cell Int 6: 11.

13. Eriksson D, Stigbrand T (2010) Radiation-induced cell death mechanisms Tumour Biol 31: 363-372.

14. Vakifahmetoglu H, Olsson M, Zhivotovsky B (2008) Death through a tragedy: mitotic catastrophe. Cell Death Differ 15: 1153-1162.

15. Golstein P, Kroemer G (2007) Cell death by necrosis: towards a molecular definition. Trends Biochem Sci 32: 37-43.

16. Wu W, Liu P, Li J (2012) Necroptosis: an emerging form of programmed cell death. Crit Rev Oncol Hematol 82: 249-258.

17. Nehs MA, Lin Cl, Kozono DE, Whang EE, Cho NL, et al. (2011) Necroptosis is a novel mechanism of radiation-induced cell death in anaplastic thyroid and adrenocortical cancers. Surgery 150: 1032-1039.

18. Mirzayans R, Scott A, Cameron M, Murray D (2005) Induction of accelerated senescence by gamma radiation in human solid tumor-derived cell lines expressing wild-type TP53. Radiat Res 163: 53-62.

19. Abend M (2003) Reasons to reconsider the significance of apoptosis for cancer therapy. Int J Radiat Biol 79: 927-941.

20. Brown JM, Attardi LD (2005) The role of apoptosis in cancer development and treatment response. Nat Rev Cancer 5: 231-237.
21. Suzuki M, Boothman DA (2008) Stress-induced premature senescence (SIPS)-influence of SIPS on radiotherapy. J Radiat Res 49: 105-112.

22. Gewirtz DA, Holt SE, Elmore LW (2008) Accelerated senescence: an emerging role in tumor cell response to chemotherapy and radiation. Biochem Pharmacol 76: $947-957$.

23. Supiot S, Shubbar S, Fleshner N, Warde P, Hersey K, et al. (2008) A phase trial of pre-operative radiotherapy for prostate cancer: clinical and translational studies. Radiother Oncol 88: 53-60.

24. Mirzayans R, Andrais B, Scott A, Murray D (2012) New insights into p53 signaling and cancer cell response to DNA damage: implications for cancer therapy. J Biomed Biotechnol 2012: 170325.

25. Mirzayans R, Murray D (2007) Cellular senescence: Implications for cancer therapy. Nova Science Publishers, Inc., Hauppauge, New York 1-64.

26. Roninson IB, Broude EV, Chang BD (2001) If not apoptosis, then what? Treatment-induced senescence and mitotic catastrophe in tumor cells. Drug Resist Updat 4: 303-313

27. Mirzayans R, Andrais B, Hansen G, Murray D (2012) Role of p16(INK4A) in Replicative Senescence and DNA Damage-Induced Premature Senescence in p53-Deficient Human Cells. Biochem Res Int 2012: 951574.

28. Wang M, Morsbach F, Sander D, Gheorghiu L, Nanda A, et al. (2011) EGF receptor inhibition radiosensitizes NSCLC cells by inducing senescence in cells sustaining DNA double-strand breaks. Cancer Res 71: 6261-6269.

29. Gudkov AV, Komarova EA (2003) The role of p53 in determining sensitivity to radiotherapy. Nat Rev Cancer 3: 117-129.

30. Luce A, Courtin A, Levalois C, Altmeyer-Morel S, Romeo PH, et al. (2009) Death receptor pathways mediate targeted and non-targeted effects of ionizing radiations in breast cancer cells. Carcinogenesis 30: 432- 439.

31. Vakifahmetoglu H, Olsson M, Tamm C, Heidari N, Orrenius S, et al. (2008) DNA damage induces two distinct modes of cell death in ovarian carcinomas. Cell Death Differ 15: 555-566

32. Gewirtz DA (2013) Autophagy and senescence in cancer therapy. J Cell Physio 229: 6-9.

33. Coppé JP, Desprez PY, Krtolica A Campisi J (2010) The senescenceassociated secretory phenotype: the dark side of tumor suppression. Annu Rev Pathol 5: 99-118.

34. Jones KR, Elmore LW, Jackson-Cook C, Demasters G, Povirk LF, et al. (2005) p53-Dependent accelerated senescence induced by ionizing radiation in breast tumour cells. Int J Radiat Biol 81: 445-458.

35. DeMasters GA, Gupta MS, Jones KR, Cabot M, Wang H, et al. (2004) Potentiation of cell killing by fractionated radiation and suppression of proliferative recovery in MCF-7 breast tumor cells by the Vitamin D3 analog EB 1089. J Steroid Biochem Mol Biol 92: 365-374.

36. Rajaraman R, Rajaraman MM, Rajaraman SR, Guernsey DL (2005) Neosis--a paradigm of self-renewal in cancer. Cell Biol Int 29: 1084-1097.

37. Roberson RS, Kussick SJ, Vallieres E, Chen SY, Wu DY (2005) Escape from therapy-induced accelerated cellular senescence in p53-null lung cancer cells and in human lung cancers. Cancer Res 65: 2795-2803.

38. Elmore LW, Di X, Dumur C, Holt SE, Gewirtz DA (2005) Evasion of a singlestep, chemotherapy-induced senescence in breast cancer cells: implications for treatment response. Clin Cancer Res 11: 2637-2643.

39. Gewirtz DA (2009) Autophagy, senescence and tumor dormancy in cancer therapy. Autophagy 5: 1232-1234.

40. Sliwinska MA, Mosieniak G, Wolanin K, Babik A, Piwocka K, et al. (2009) Induction of senescence with doxorubicin leads to increased genomic instability of HCT116 cells. Mech Ageing Dev 130: 24-32.

41. Wu PC, Wang Q, Grobman L, Chu E, Wu DY (2012) Accelerated cellular senescence in solid tumor therapy. Exp Oncol 34: 298-305.

42. Ewald JA, Jarrard DF (2012) Decreased skp2 expression is necessary but not sufficient for therapy-induced senescence in prostate cancer. Transl Oncol 5 : 278-287.

43. Wang Q, Wu PC, Dong DZ, Ivanova I, Chu E, et al. (2013) Polyploidy road to therapy-induced cellular senescence and escape. Int J Cancer 132: 15051515. 
Citation: Murray D, Mirzayans R (2013) Role of Therapy-Induced Cellular Senescence in Tumor Cells and its Modification in Radiotherapy: The Good, The Bad and The Ugly. J Nucl Med Radiat Ther S6: 018. doi:10.4172/2155-9619.S6-018

44. Tsai KK, Stuart J, Chuang YY, Little JB, Yuan ZM (2009) Low-dose radiationinduced senescent stromal fibroblasts render nearby breast cancer cells radioresistant. Radiat Res 172: 306-313.

45. Canino C, Mori F, Cambria A, Diamantini A, Germoni S, et al. (2012) SASP mediates chemoresistance and tumor-initiating-activity of mesothelioma cells. Oncogene 31: 3148-3163.

46. Laberge RM, Awad P, Campisi J, Desprez PY (2012) Epithelial-mesenchymal transition induced by senescent fibroblasts. Cancer Microenviron 5: 39-44.

47. Basu D, Reyes-Mugica M, Rebbaa A (2012) Role of the beta catenin destruction complex in mediating chemotherapy-induced senescence-associated secretory phenotype. PLoS One 7: e52188.

48. Passos JF, Nelson G, Wang C, Richter T, Simillion C, et al. (2010) Feedback between $\mathrm{p} 21$ and reactive oxygen production is necessary for cell senescence. Mol Syst Biol 6: 347.

49. Skinner HD, Sandulache VC, Ow TJ, Meyn RE, Yordy JS, et al. (2012) TP53 disruptive mutations lead to head and neck cancer treatment failure through inhibition of radiation-induced senescence. Clin Cancer Res 18: 290-300.

50. Wang Q, Wu PC, Roberson RS, Luk BV, Ivanova I, et al. (2011) Survivin and escaping in therapy-induced cellular senescence. Int J Cancer 128: 1546-1558.

51. Bromfield GP, Meng A, Warde P, Bristow RG (2003) Cell death in irradiated prostate epithelial cells: role of apoptotic and clonogenic cell kill. Prostate Cancer Prostatic Dis 6: 73-85.

52. Lehmann BD, McCubrey JA, Jefferson HS, Paine MS, Chappell WH, et al. (2007) A dominant role for p53-dependent cellular senescence in radiosensitization of human prostate cancer cells. Cell Cycle 6: 595-605.

53. Sidi R, Pasello G, Opitz I, Soltermann A, Tutic M, et al. (2011) Induction of senescence markers after neo-adjuvant chemotherapy of malignant pleural mesothelioma and association with clinical outcome: an exploratory analysis. Eur J Cancer 47: 326-332.
54. Park D, Magis AT, Li R, Owonikoko TK, Sica GL, et al. (2013) Novel smallmolecule inhibitors of Bcl-XL to treat lung cancer. Cancer Res 73: 5485-5496.

55. Luo H, Yount C, Lang H, Yang A, Riemer EC, et al. (2013) Activation of p53 with Nutlin-3a radiosensitizes lung cancer cells via enhancing radiation-induced premature senescence. Lung Cancer 81: 167-173.

56. Arya AK, El-Fert A, Devling T, Eccles RM, Aslam MA, et al. (2010) Nutlin-3, the small-molecule inhibitor of MDM2, promotes senescence and radiosensitises laryngeal carcinoma cells harbouring wild-type p53. Br J Cancer 103: 186-195.

57. Han NK, Kim BC, Lee HC, Lee YJ, Park MJ, et al. (2012) Secretome analysis of ionizing radiation-induced senescent cancer cells reveals that secreted RKIP plays a critical role in neighboring cell migration. Proteomics 12: 2822-2832.

58. Tasdemir N, Lowe SW (2013) Senescent cells spread the word: non-cell autonomous propagation of cellular senescence. EMBO J 32: 1975-1976.

59. Murray D, McEwan AJ (2007) Radiobiology of systemic radiation therapy. Cancer Biother Radiopharm 22: 1-23.

60. Zwicker RD, Meigooni A, Mohiuddin M (2004) Therapeutic advantage of grid irradiation for large single fractions. Int J Radiat Oncol Biol Phys 58: 1309-1315.

61. Dilmanian FA, Morris GM, Zhong N, Bacarian T, Hainfeld JF, et al. (2003) Murine EMT-6 carcinoma: high therapeutic efficacy of microbeam radiation therapy. Radiat Res 159: 632-641.

62. Kishikawa H, Wang K, Adelstein SJ, Kassis Al (2006) Inhibitory and stimulatory bystander effects are differentially induced by lodine-125 and lodine-123. Radiat Res 165: 688-694.

63. Kapty J, Murray D, Mercer J (2010) Radiotracers for noninvasive molecular imaging of tumor cell death. Cancer Biother Radiopharm 25: 615-628.

64. Bonzon C, Droin NM, Maurer U, Ricci JE (2002) Death in paradise. Cell Death Differ 9: 780-782.
This article was originally published in a special issue, Cancer Radiation Therapy handled by Editor(s). Dr. Xin Chen, University of Arkansas for Medical Sciences, USA 